\title{
Echocardiographic Characteristics of Subjects With COVID-19: A Case Series
}

\author{
Raymundo Vera-Pineda ${ }^{\mathrm{a}}$, Edgar Francisco Carrizales-Sepulveda ${ }^{\mathrm{a}}$, Adrian Camacho-Ortiz ${ }^{\mathrm{b}}$, \\ Laura Nuzzolo-Shihadeh ${ }^{\mathrm{b}}$, Francisco Cruz-Ramos ${ }^{\mathrm{c}}$, Alejandro Ordaz-Farias ${ }^{\mathrm{c}}$, \\ Mario Alberto Benavides-Gonzalez ${ }^{\text {a }}$, Gabriel Carranza-Villegas ${ }^{\text {a,d }}$
}

\begin{abstract}
Although coronavirus disease 2019 (COVID-19) manifests in most cases with respiratory symptoms, other presentations can occur. Direct damage to the cardiovascular system has been reported and recently, acute myocardial injury has been identified as a risk factor for mortality. Transthoracic echocardiography is a non-invasive tool that allows the detection of myocardial damage with validated markers (left ventricular ejection fraction and global longitudinal strain). Herein, we present the echocardiographic findings in four patients with COVID-19. All cases had acute respiratory distress syndrome $(100 \%)$. Three out of four had elevated levels of creatine kinase and creatine kinase myocardial band. One case had ventricular concentric remodeling $(25 \%)$. All cases $(100 \%)$ had altered ventricular function: two had a reduced ejection fraction (50\%) and, of those available for global longitudinal strain analysis, all had abnormal global longitudinal strain $(100 \%)$. One case was found to have a tricuspid vegetation of $12 \times 10 \mathrm{~mm}$ with no other manifestation of endocarditis. All of our cases had left ventricular dysfunction as assessed by echocardiography. One of our patients had a vegetation in the tricuspid valve. Two of our cases had a reduced ejection fraction. The importance of acute cardiac injury in COVID-19 has recently been established. A recent study found it to be an independent risk factor for mortality in patients with this disease. Information regarding echocardiographic characteristics of this population is scarce. Further research to elucidate the impact of these characteristics on morbidity and mortality is urgently needed.
\end{abstract}

Keywords: COVID-19; Heart failure; Transthoracic echocardiography

Manuscript submitted April 28, 2020, accepted May 8, 2020

Published online June 3, 2020

${ }^{a}$ Cardiology Department, Hospital Universitario, Universidad Autonoma de Nuevo Leon (UANL), Nuevo Leon, Mexico

bInfectious Diseases Department, Hospital Universitario, Universidad Autonoma de Nuevo Leon (UANL), Nuevo Leon, Mexico

'Echocardiography Laboratory, Cardiology Department, Hospital Universitario, Universidad Autonoma de Nuevo Leon (UANL), Nuevo Leon, Mexico ${ }^{\mathrm{d} C o r r e s p o n d i n g ~ A u t h o r: ~ G a b r i e l ~ C a r r a n z a-V i l l e g a s, ~ C a r d i o l o g y ~ D e p a r t m e n t, ~}$ Hospital Universitario, Universidad Autonoma de Nuevo Leon, Monterrey, Nuevo Leon, Mexico. Email: carranzavillegas@gmail.com

doi: https://doi.org/10.14740/cr1084

\section{Introduction}

In December 2019, a wave of cases of atypical pneumonia emerged from Wuhan City, Hubei Province in China [1, 2]. This disease (coronavirus disease 2019 (COVID-19)) is caused by the beta coronavirus severe acute respiratory syndrome coronavirus 2 (SARS-CoV-2) and has rapidly spread around the world, being declared a pandemic since March 11. Early reports suggest that COVID-19 is generally mild; nevertheless, up to $19 \%$ of cases will require in-hospital management, and $6 \%$ will need to be admitted to the intensive care unit (ICU) [3]. Although COVID-19 manifests in most patients with respiratory symptoms, other presentations can occur, and it has been suggested that there could be direct damage to the cardiovascular system [4-6]. In addition, increasing concern exists regarding the severity of the disease in patients with prior cardiovascular disease (CVD), since this group has a worse prognosis $[7,8]$. Transthoracic echocardiography (TTE) allows us to detect subtle myocardial damage with tools that are associated with prognosis, e.g. left ventricular ejection fraction (LVEF) or global longitudinal strain (GLS) [9]. Herein, we present the echocardiographic findings in four patients with COVID-19.

\section{Case Reports}

We included four cases in this series. All were evaluated between March and April 2020 at the "Dr. Jose Eleuterio Gonzalez", University Hospital in Monterrey, Mexico. Here, subjects with a suspicion of infection were evaluated by an infectiologist and those deemed at risk were tested using a real-time reverse transcription-polymerase chain reaction (RTPCR). Subjects with positive results were then admitted to a special ward designated for management of these subjects. To ensure subjects' safety, we followed the Ethical Guidelines of the 1975 Declaration of Helsinki and obtained verbal consent from patients' next of kin. The cases were classified as severe $(n=1)$ or critical $(n=3)$ [10]. A certified echocardiographer performed the TTEs wearing personal protective equipment (PPE) and using the current American Society of Echocardiography (ASE) guidelines for image acquisition and interpretation. We also used the current ASE guidelines to obtain normal reference values $[11,12]$. 
Table 1. Baseline Clinical and Laboratory Characteristics

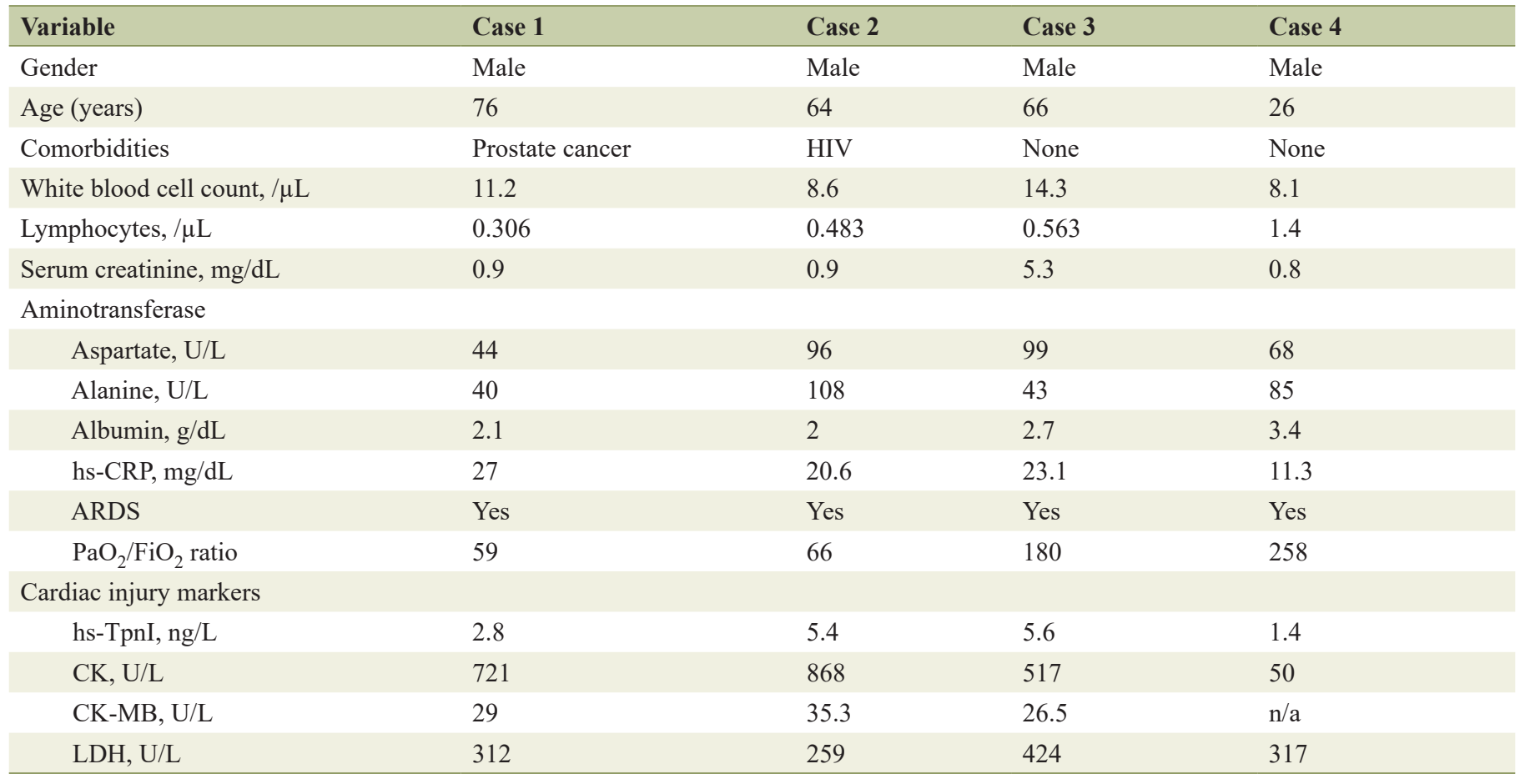

hs-CRP: high-sensitivity C-reactive protein; ARDS: acute respiratory distress syndrome; hs-Tpnl: high-sensitivity troponin I; CK: creatine kinase; CKMB: creatine kinase myocardial band; LDH: lactate dehydrogenase.

\section{Case 1}

A 76-year-old man presented to the emergency room (ER) with a 10-day history of shortness of breath, fever and dry cough. He had a previous diagnosis of prostate cancer, currently in remittance. His baseline characteristics are shown in Table 1. He had leukopenia, elevated cardiac markers including creatine kinase $(\mathrm{CK})$, creatine kinase myocardial band (CK-MB) and lactate dehydrogenase $(\mathrm{LDH})$ and a severe acute respiratory distress syndrome (ARDS) with a $\mathrm{PaO}_{2} / \mathrm{FiO}_{2}$ ratio of 59, which was initially treated with a high-flow nasal cannula (HFNC) and supportive treatment. A TTE was performed during day 8 of his hospital stay. This revealed abnormal LV function with an LVEF of $35 \%$ and a GLS of $-14 \%$. Echocardiographic characteristics are shown in Table 2. He was intubated the next day and developed barotrauma with a right pneumothorax that required a pleural catheter for evacuation. Afterward, on his 10th day, he developed an acute kidney injury (AKI) with anuria, multiorgan failure and acidosis. He passed away on his 11th day.

\section{Case 2}

A 64-year-old man who was human immunodeficiency virus (HIV)-positive presented to the ER with a 4-day history of headache and malaise. On interrogation, he referred traveling to the United States during the previous 2 weeks. Baseline characteristics are shown in Table 1. On admittance, he had a temperature of $38.3^{\circ} \mathrm{C}$. On his seventh day of hospital stay, he developed respiratory insufficiency and was intubated. Septic shock was diagnosed on the eighth day and vasopressor support was initiated. A TTE was done to evaluate shock etiology, which showed an abnormal ventricular geometry with concentric remodeling and a GLS of $-18 \%$ with an LVEF of $66 \%$ (Table 2). During his time in the ICU, on day 14, the subject developed new infiltrates on his chest X-ray, so a diagnosis of ventilator-associated pneumonia was entertained, and broadspectrum antibiotics were started. This was eventually found to be caused by Klebsiella spp, according to an endotracheal aspiration culture. On his 21 st day, he presented massive hemoptysis and expired.

\section{Case 3}

A 66-year-old man presented with a 5-day history of shortness of breath, fever and dry cough. His previous medical history was unremarkable. He reported having a family member that had traveled to the United States during the previous month. He presented to the ER with respiratory insufficiency syndrome. During his first day in the ICU, he was classified as a moderate ARDS with a $\mathrm{PaO}_{2} / \mathrm{FiO}_{2}$ ratio of 180 (Table 1). $\mathrm{He}$ was initially managed with HFNC and, due to an altered mental state, was then intubated using video laryngoscopy. On his third day, he developed an AKI with anuria and septic shock that was treated with vasopressors. On his fourth day, prolonged intermittent renal replacement therapy was started using a non-tunneled dialysis catheter. A TTE was done on his 
Table 2. Echocardiographic characteristics and Recommended Reference Values

\begin{tabular}{|c|c|c|c|c|c|}
\hline Variable & Case 1 & Case 2 & Case 3 & Case 4 & Reference value \\
\hline LV septal thickness, $\mathrm{cm}$ & 0.8 & 1.2 & 1.1 & 0.7 & $0.6-1$ \\
\hline Posterior wall thickness, $\mathrm{cm}$ & 0.8 & 1.1 & 0.8 & 0.9 & $0.6-1$ \\
\hline Relative wall thickness & 0.31 & 0.47 & 0.32 & 0.37 & $0.24-0.42$ \\
\hline VfdVI MOD BP & 38 & 76 & $\mathrm{n} / \mathrm{a}$ & 83 & $62-150$ \\
\hline LVEF, \% & 35 & 66 & 65 & 33 & $52-72$ \\
\hline Left atrial indexed volume, $\mathrm{mL} / \mathrm{m}^{2}$ & 21.8 & 15.4 & & 11.1 & $<34$ \\
\hline Average GLS, \% & -14 & -18 & $\mathrm{n} / \mathrm{a}$ & -14 & $<-18.5 \%$ \\
\hline Aplax GLS, \% & -12 & -18 & $\mathrm{n} / \mathrm{a}$ & -14 & $<-18.5 \%$ \\
\hline a4C GLS, \% & -13 & -18 & $\mathrm{n} / \mathrm{a}$ & -13 & $<-18.5 \%$ \\
\hline $\mathrm{RV}$ basal diameter, $\mathrm{mm}$ & 33 & 34 & 47 & 39 & $25-41$ \\
\hline RV mid-cavity diameter, $\mathrm{mm}$ & 29 & 22 & 45 & 34 & $19-35$ \\
\hline MV E wave velocity, $\mathrm{m} / \mathrm{s}$ & 0.5 & 0.83 & 0.54 & 0.38 & $>50$ \\
\hline MV deceleration time, $\mathrm{ms}$ & 210 & 218 & 185 & 147 & $>200$ \\
\hline $\mathrm{E} / \mathrm{A}$ ratio & 0.63 & 1.03 & 0.69 & 0.44 & $\geq 0.8$ \\
\hline Septal e' velocity, m/s & 0.05 & 0.08 & 0.07 & 0.1 & $>7$ \\
\hline Lateral $\mathrm{e}^{\prime}$ velocity, $\mathrm{m} / \mathrm{s}$ & 0.1 & 0.09 & 0.1 & 0.1 & $>10$ \\
\hline $\mathrm{E} / \mathrm{e}^{\prime}$ ratio & 6.6 & 9.66 & 6.27 & 3.78 & $<10$ \\
\hline
\end{tabular}

Data are shown as frequencies (\%) and median (min. - max.) or mean \pm SD. LV: left ventricular; LVEF: LV ejection fraction; GLS: global longitudinal strain; a4C: apical four-chamber view; APLAX: apical long axis view; a2C: apical two-chamber view; TAPSE: tricuspid annulus plane systolic excursion; RV: right ventricular; MV: mitral valve; n/a: not available.

eighth day, showing an LVEF of $65 \%$ (Table 2), a mobile vegetation with a size of $12 \times 10 \mathrm{~mm}$ that was attached to the septal leaflet of the tricuspid valve, and severe tricuspid regurgitation (Fig. 1a). Four consecutive blood cultures were performed and all were negative. No other of Duke's criteria for infectious endocarditis were present. On his 14th day in the ICU, fever was again identified, accompanied by a new infiltrate on his chest X-ray. A diagnosis of ventilator-associated pneumonia was established and broad-spectrum antibiotics were started. At this time, a new RT-PCR for SARS-CoV-2 was performed, which was still positive. During his 22nd day in the ICU, the patient was still intubated, dependent of renal replacement therapy (RRT) and vasopressor support, with altered mentation (unarousable) during his unsuccessful ventilation weaning trials. He was being considered for an electroencephalogram to evaluate his mental status and a tracheostomy because of his prolonged intubation.

\section{Case 4}

A 26-year-old man presented to our institution with a 5-day history of malaise, fever, arthralgias, myalgias and shortness of breath. His medical background was unremarkable. On arrival, he had tachypnea and a temperature of $39.6{ }^{\circ} \mathrm{C}$. On his second day, he required supplementary oxygen through a standard nasal cannula. His laboratory results showed hypoalbuminemia and elevated LDH (Table 1). On his third day, a TTE was performed, which showed abnormal regional function with hypokinetic segments in the basal and medial inferior and inferolateral territories, an LVEF of $33 \%$ and a GLS of $-14 \%$ (Table 2). We present the GLS pattern of this patient in Figure 1b. This subject was managed with supportive treatment and supplementary oxygen, which has finally removed during his ninth day in the hospital. He was discharged on day 13.

\section{Discussion}

We found several cardiac manifestations in subjects with confirmed COVID-19. One subject had ventricular concentric remodeling, one had a tricuspid vegetation and two heart failure with a reduced ejection fraction (one was a young patient with no other comorbidity). Also, subjects available for GLS analysis showed signs of ventricular dysfunction.

Several recent studies have explored the relationship be- 


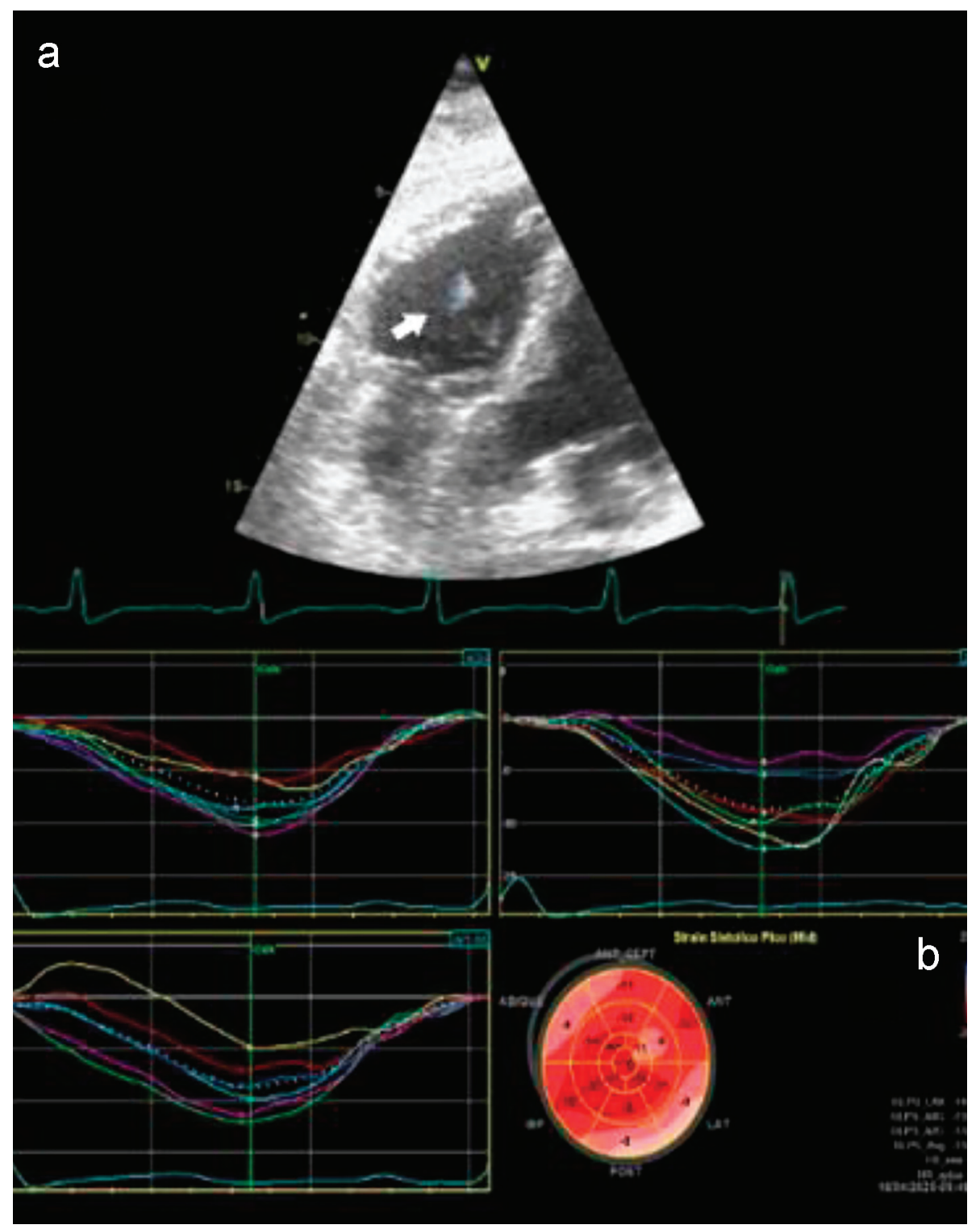

Figure 1. (a) Modified a4c view showing the tricuspid vegetation of case 3. (b) Peak GLS showing decreased values in the inferolateral and inferoseptal territories of case 4. a4C: apical four-chamber view; GLS: global longitudinal strain.

tween COVID-19 and myocardial damage. Two recent studies have reported increased mortality in subjects with an elevation in hs-troponins, and have proposed cardiac injury as an independent risk factor for mortality (hazard ratio (HR): 4.26) [5, 6]. Importantly, the study by Guo et al [5] also showed that elevations in cardiac troponins were associated with higher $\mathrm{C}$-reactive protein (CRP), D-dimer, cytokines and N-terminal pro-B-type natriuretic peptide (NT-proBNP) levels, suggesting there might be a link between myocardial injury, inflammation and ventricular dysfunction. None of these trials have reported echocardiographic evidence of myocardial dysfunction. Similar to other reports, our subjects had normal baseline levels of hs-troponins.

In this series, serial measurements of troponins or D-dimer determination were not available, but increased levels of other cardiac markers such as CK, CK-MB and myocardial dysfunction detected by TTE were found. None of our patients had previous known CVD. Therefore, myocardial dysfunction could represent a direct manifestation of COVID-19, and also myocardial abnormalities seen in critically ill patients [13]. One of our patients had a tricuspid vegetation, severe tricuspid regurgitation and repeated negative blood cultures. Interestingly, previous reports in subjects with SARS-CoV infection show the presence of endocarditis with marantic vegetations [14]. Recently, the presence of a COVID-19-associated coagulopathy (CAC) characterized by hypercoagulability has been proposed, and recent studies have found a presence of thrombotic events in $31 \%$ of subjects with COVID-19, mainly pulmonary embolism $[15,16]$. We were unable to perform a pathologic evaluation of our subject's vegetation for this report, and computed tomography (CT)-pulmonary angiography was 
not available because of his clinical condition. Although all of our subjects were receiving thromboprophylaxis, the possibility of this being a thrombus attached to the valve remains.

Currently, the impact on the prognosis of a diminished LVEF or LV GLS in subjects with COVID-19 is not clear. A recent study in a cohort of 120 subjects with COVID-19, found that tricuspid annular plane systolic excursion (TAPSE) and right ventricular GLS were significantly related to mortality, even though the values found in deceased subjects were within normal reference values, like the patients here presented [17]. No information is presented about LV GLS. More research is urgently needed to elucidate the pathophysiological affection of the cardiovascular system in COVID-19. Tools for the detection of high-risk subjects for management and resource allocation are vital, considering the strain of health systems because of the COVID-19 pandemic.

This report has several limitations. First, image acquisition in these subjects is difficult: PPE is required to minimize exposure of all the personnel involved, and all equipments need to be sterilized between studies. Currently, the use of TTE must be evaluated on a case-by-case basis at every location. Second, we were not able to obtain D-dimer values or CT for our subjects. Also, our sample size is small. Finally, even though none of our subjects had previous known CVD, some of the findings we report could predate the acquisition of COVID-19. However, amid this pandemic, the data are of value.

In conclusion, all of our subjects showed an altered LV function, assessed by LVEF and GLS. Subjects with COVID-19 present several types of myocardial dysfunction assessed by TTE, even in the absence of prior CVD.

\section{Acknowledgments}

We would like to thank Dr. Sergio Lozano-Rodriguez for the critical evaluation and edition of this manuscript.

\section{Financial Disclosure}

None to declare.

\section{Conflict of Interest}

None to declare.

\section{Informed Consent}

Verbal consent was obtained from subjects' next of kin. We present only de-identified data to ensure patients' anonymity is preserved.

\section{Author Contributions}

RVP: partial role in protocol design; partial contribution in data acquisition and manuscript elaboration; equal role in manuscript edition. EFCS: partial contribution in data acquisition and manuscript elaboration; equal role in manuscript edition. ACO: partial role in protocol design; partial in manuscript elaboration; equal role in manuscript edition. LNS: partial role in protocol design; partial contribution in data acquisition and manuscript elaboration; equal role in manuscript edition. FCR: partial contribution in data acquisition and manuscript elaboration; equal role in manuscript edition. AOF: main role in protocol design; partial contribution in data acquisition and manuscript elaboration; equal role in manuscript edition. MABG: main role in protocol design; partial contribution in data acquisition and manuscript elaboration; equal role in manuscript edition. GCV: main contribution in image acquisition and interpretation; partial role in manuscript elaboration; equal role in manuscript edition.

\section{Data Availability}

The data supporting the findings of this study are available from the corresponding author upon reasonable request.

\section{References}

1. Wang D, Hu B, Hu C, Zhu F, Liu X, Zhang J, Wang B, et al. Clinical characteristics of 138 hospitalized patients with 2019 novel coronavirus-infected pneumonia in Wuhan, China. JAMA. 2020;323(11):1061-1069.

2. Zhou P, Yang XL, Wang XG, Hu B, Zhang L, Zhang W, Si HR, et al. A pneumonia outbreak associated with a new coronavirus of probable bat origin. Nature. 2020;579(7798):270-273.

3. $\mathrm{Wu} \mathrm{Z}, \mathrm{McGoogan}$ JM. Characteristics of and important lessons from the coronavirus disease 2019 (COVID-19) outbreak in China: summary of a report of 72314 cases from the Chinese Center for Disease Control and Prevention. JAMA. 2020;323(13):1239-1242.

4. Huang C, Wang Y, Li X, Ren L, Zhao J, Hu Y, Zhang $\mathrm{L}$, et al. Clinical features of patients infected with 2019 novel coronavirus in Wuhan, China. Lancet. 2020;395(10223):497-506.

5. Guo T, Fan Y, Chen M, Wu X, Zhang L, He T, Wang H, et al. Cardiovascular implications of fatal outcomes of patients with coronavirus disease 2019 (COVID-19). JAMA Cardiol. 2020.

6. Shi S, Qin M, Shen B, Cai Y, Liu T, Yang F, Gong W, et al. Association of cardiac injury with mortality in hospitalized patients with COVID-19 in Wuhan, China. JAMA Cardiol. 2020.

7. Bonow RO, Fonarow GC, O'Gara PT, Yancy CW. Association of coronavirus disease 2019 (COVID-19) with myocardial injury and mortality. JAMA Cardiol. 2020.

8. Zheng YY, Ma YT, Zhang JY, Xie X. COVID-19 and the cardiovascular system. Nat Rev Cardiol. 2020;17(5):259260.

9. Zito C, Longobardo L, Citro R, Galderisi M, Oreto L, Carerj ML, Manganaro R, et al. Ten years of 2D longitu- 
dinal strain for early myocardial dysfunction detection: a clinical overview. Biomed Res Int. 2018;2018:8979407.

10. Interim clinical guidance for management of patients with confirmed coronavirus disease (COVID-19). 2020. Available at: https://www.cdc.gov/coronavirus/2019ncov/hcp/clinical-guidance-management-patients.html. Accessed on April 14, 2020.10.7175/cmi.v14i1.1467.

11. Lang RM, Badano LP, Mor-Avi V, Afilalo J, Armstrong A, Ernande L, Flachskampf FA, et al. Recommendations for cardiac chamber quantification by echocardiography in adults: an update from the American Society of Echocardiography and the European Association of Cardiovascular Imaging. J Am Soc Echocardiogr. 2015;28(1):139 e14.

12. Marwick TH, Leano RL, Brown J, Sun JP, Hoffmann R, Lysyansky P, Becker M, et al. Myocardial strain measurement with 2-dimensional speckle-tracking echocardiography: definition of normal range. JACC Cardiovasc Imaging. 2009;2(1):80-84.

13. Frencken JF, van Baal L, Kappen TH, Donker DW, Horn
J, van der Poll T, van Klei WA, et al. Myocardial injury in critically ill patients with community-acquired pneumonia. A cohort study. Ann Am Thorac Soc. 2019;16(5):606612.

14. Chong PY, Chui P, Ling AE, Franks TJ, Tai DY, Leo YS, Kaw GJ, et al. Analysis of deaths during the severe acute respiratory syndrome (SARS) epidemic in Singapore: challenges in determining a SARS diagnosis. Arch Pathol Lab Med. 2004;128(2):195-204.

15. Klok FA, Kruip M, van der Meer NJM, Arbous MS, Gommers D, Kant KM, Kaptein FHJ, et al. Incidence of thrombotic complications in critically ill ICU patients with COVID-19. Thromb Res. 2020.

16. Connors JM, Levy JH. Thromboinflammation and the hypercoagulability of COVID-19. J Thromb Haemost. 2020.

17. Li Y, Li H, Zhu S, Xie Y, Wang B, He L, Zhang D, et al. Prognostic value of right ventricular longitudinal strain in patients with COVID-19. JACC: Cardiovascular Imaging. 2020:3423. 